\title{
Effect of the bound nucleon form factors on charged-current neutrino-nucleus scattering
}

\author{
K. Tsushima, ${ }^{1, *}$ Hungchong Kim, ${ }^{2, \dagger}$ and K. Saito ${ }^{3, *}$ \\ ${ }^{1}$ Department of Physics and Astronomy, University of Georgia, Athens, Georgia 30602, USA \\ ${ }^{2}$ Institute of Physics and Applied Physics, Yonsei University, Seoul 120-749, Korea \\ ${ }^{3}$ Department of Physics, Faculty of Science and Technology, Tokyo University of Science, Noda 278-8510, Japan
}

(Received 9 March 2004; published 22 September 2004)

\begin{abstract}
We study the effect of bound nucleon form factors on charged-current neutrino-nucleus scattering. The bound nucleon form factors of the vector and axial-vector currents are calculated in the quark-meson coupling model. We compute the inclusive ${ }^{12} \mathrm{C}\left(\nu_{\mu}, \mu^{-}\right) X$ cross sections using a relativistic Fermi gas model with the calculated bound nucleon form factors. The effect of the bound nucleon form factors for this reaction is a reduction of $\sim 8 \%$ for the total cross section, relative to that calculated with the free nucleon form factors.
\end{abstract}

DOI: 10.1103/PhysRevC.70.038501

There has been considerable interest in possible changes in the bound nucleon properties [1]. A number of evidences, such as the nuclear European muon collaboration (EMC) effect [2], the quenching [3,4] (enhancing [5]) of the space (time) component of the effective one-body axial coupling constant in nuclear $\beta$ decays, the missing strength of the response functions in nuclear inelastic electron scattering, and the suppression of the Coulomb sum rule [6], have stimulated investigations of whether or not the quark degrees of freedom play any vital role.

Recently, the electromagnetic form factors of bound protons were studied in polarized $\left(\vec{e}, e^{\prime} \vec{p}\right)$ scattering experiments on ${ }^{16} \mathrm{O}$ and ${ }^{4} \mathrm{He}$ [7]. The results from MAMI and Jefferson Lab on ${ }^{4} \mathrm{He}[7]$ concluded that ratio of the electric $\left(G_{E}^{p}\right)$ to magnetic $\left(G_{M}^{p}\right)$ Sachs proton form factors differs by $\sim 10 \%$ in ${ }^{4} \mathrm{He}$ from that in ${ }^{1} \mathrm{H}$. Conventional models employing free proton form factors, phenomenological optical potentials, and bound state wave functions, as well as relativistic corrections, meson exchange currents (MEC), isobar contributions, and final state interactions $[7,8]$, all fail to account for the observed effect in ${ }^{4} \mathrm{He}$ [7]. Indeed, full agreement with the data was obtained only when, in addition to these standard nuclear-structure corrections, a small correction due to the internal structure of the bound proton was taken into account $[7,9]$.

Here, we study the effect of the bound nucleon form factors on neutrino-nucleus scattering. ${ }^{1}$ As an example, we compute the inclusive ${ }^{12} \mathrm{C}\left(\nu_{\mu}, \mu^{-}\right) X$ cross sections that have been measured by the LSND collaboration [10]. It is known that the existing calculations for the total cross section based on the nucleon and meson degrees of freedom overestimate the data by $\sim 30 \%$ to $\sim 100 \%[10,11]$. Because our aim is to focus on the effects due to the internal structure change of the bound nucleon, we use a relativistic Fermi gas model

\footnotetext{
*Present address: IFT-UNESP, Rua Pamplona 145, 01405-900, São Paulo-SP, Brazil. Email address: tsushima@ift.unesp.br

${ }^{\dagger}$ Email address: hung@phya.yonsei.ac.kr

${ }^{\ddagger}$ Email address: ksaito@ph.noda.tus.ac.jp

${ }^{1}$ Because the renormalization of axial-vector form factors are the same for the time and space components in this study (quenched), we will not discuss the time component.
}

PACS number(s): 13.40.Gp, 25.30.Pt, 12.39.Ba, 21.65.+f

$[12,13]$, which is simple and transparent for the purpose, while implementing the bound nucleon form factors calculated in the quark-meson coupling (QMC) model $[9,14]$. Thus, we do not include the other nuclear structure corrections $[11,15]$.

Of course, it is difficult to separate exactly the effects we consider here from the standard nuclear-structure corrections, particularly from MEC. However, since the relevant current operators in this study are one-body quark (pion) operators acting on the quarks (pion cloud) in the nucleon, a double counting with the model-dependent MEC $[4,16]$ (the current operators act on the exchanged mesons) is expected to be avoided. The same is also true for the modelindependent meson pair currents, because they are based on the antinucleon degrees of freedom $[3,4,16]$. For the vector current, a double counting with MEC may be practically avoided as the analyses for the ${ }^{4} \mathrm{He}\left(\vec{e}, e^{\prime} \vec{p}\right)^{3} \mathrm{H}$ experiments [7] have shown. For the axial-vector current, the quenching of the axial coupling constant $\left[g_{A}=G_{A}(0)\right]$ due to the modelindependent meson pair currents was estimated [4] using a Fermi gas model. The quenching due to the pair currents amounts to a $2 \%$ at normal nuclear matter density, thus contributing negligibly to the cross section. Hence, the double counting from the interference between the axial-vector and vector currents is also expected to be small when considering the analyses for the ${ }^{4} \mathrm{He}\left(\vec{e}, e^{\prime} \vec{p}\right)^{3} \mathrm{H}$ experiments. Thus, the effect we consider here, which originates from the change of the internal quark wave function, is additional to the standard nuclear-structure corrections.

The QMC model [17] has been successfully applied to many problems of nuclear physics and hadronic properties in nuclear medium [18]. In the model, the medium effects arise through the self-consistent coupling of scalar $(\sigma)$ and vector $(\omega)$ meson fields to confined quarks rather than to the nucleons. As a result, the internal structure of the bound nucleon is modified by the surrounding nuclear medium. (Details of the QMC model are given in Refs. [17,18].)

Assuming $G$ parity (no second-class current), the chargedcurrent vector and axial form factors for free nucleons with mass $m_{N}$ are defined by

$$
\begin{aligned}
\left\langle p^{\prime} s^{\prime}\left|V_{a}^{\mu}(0)\right| p s\right\rangle= & \bar{u}_{s^{\prime}}\left(p^{\prime}\right)\left\{F_{1}\left(Q^{2}\right) \gamma^{\mu}+i\left[F_{2}\left(Q^{2}\right) / 2 m_{N}\right] \sigma^{\mu \nu}\right. \\
& \left.\times\left(p^{\prime}-p\right)_{\nu}\right\}\left(\tau_{a} / 2\right) u_{s}(p),
\end{aligned}
$$




$$
\begin{aligned}
\left\langle p^{\prime} s^{\prime}\left|A_{a}^{\mu}(0)\right| p s\right\rangle= & \bar{u}_{s^{\prime}}\left(p^{\prime}\right)\left\{G_{A}\left(Q^{2}\right) \gamma^{\mu}+\left[G_{P}\left(Q^{2}\right) / 2 m_{N}\right]\right. \\
& \left.\times\left(p^{\prime}-p\right)^{\mu}\right\} \gamma_{5}\left(\tau_{a} / 2\right) u_{s}(p),
\end{aligned}
$$

where $Q^{2} \equiv-\left(p^{\prime}-p\right)^{2}$, and other notations should be selfexplanatory. The vector form factors, $F_{1}\left(Q^{2}\right)$ and $F_{2}\left(Q^{2}\right)$, are related to the electric $\left[G_{E}\left(Q^{2}\right)\right]$ and magnetic $\left[G_{M}\left(Q^{2}\right)\right] \mathrm{Sa}$ chs form factors by the conserved vector current hypothesis. The induced pseudoscalar form factor, $G_{P}\left(Q^{2}\right)$, is dominated by the pion pole and can be calculated using the partially conserved axial vector current (PCAC) relation [19]. Nevertheless, the contribution from $G_{P}\left(Q^{2}\right)$ to the cross section is proportional to (lepton mass) $)^{2} / m_{N}^{2}$, and is small in the present study. We note that since there is another vector in the nuclear medium, the nuclear (matter) four-velocity, there may arise various other form factors in addition to those in Eqs. (1) and (2). The modification of the nucleon internal structure studied here may also be expected to contribute to such form factors. However, at this stage, information on such form factors is very limited and not well under control theoretically and experimentally. Thus, we focus on the inmedium changes of the free form factors given in Eqs. (1) and (2), and study their effects on neutrino-nucleus scattering. (Hereafter we denote the in-medium quantities by an asterisk.)

Using the improved cloudy bag model (ICBM) [20] and QMC, the electromagnetic and axial form factors in nuclear medium are calculated in the Breit frame $[9,14]$

$$
G_{E, M, A}^{Q M C^{*}}\left(Q^{2}\right)=\eta^{2} G_{E, M, A}^{\mathrm{sph}{ }^{*}}\left(\eta^{2} Q^{2}\right),
$$

where $\eta=\left(m_{N}^{*} / E_{N}^{*}\right)$ is the scaling factor with $E_{N}^{*}$ $=\sqrt{m_{N}^{* 2}+Q^{2} / 4}$ the energy, and $m_{N}^{*}$ the effective nucleon mass in nuclear medium. The explicit expressions for Eq. (3) are given in Refs. [9,14]. The ICBM includes a Peierls-Thouless projection to account for center of mass and recoil corrections, and a Lorentz contraction of the internal quark wave function $[20,21]$.

Now we calculate the ratios of the bound to free nucleon form factors, $\left[G_{E, M, A}^{Q M C^{*}} / G_{E, M, A}^{I C B M}\right.$ free $]$, to estimate the bound nucleon form factors. Using the empirical parametrizations in free space $G_{E, M, A}^{e m p}[22,23]$, the bound nucleon form factors $G_{E, M, A}^{*}$ are calculated by

$G_{E, M, A}^{*}\left(Q^{2}\right)=\left[G_{E, M, A}^{Q M C^{*}}\left(Q^{2}\right) / G_{E, M, A}^{I C B M}\right.$ free $\left.\left(Q^{2}\right)\right] G_{E, M, A}^{e m p}\left(Q^{2}\right)$.

Note that the pion cloud effect is not included in the axial form factor in the present treatment [14]. However, the normalized $Q^{2}$ dependence [divided by $g_{A}=G_{A}(0)$ ] reproduces relatively well the empirical parametrization [14]. Furthermore, the relative modification of $G_{A}^{*}\left(Q^{2}\right)$ due to the pion cloud is expected to be small since the pion cloud contribution to entire $g_{A}$ is $\sim 8 \%$ [19] without a specific center-ofmass correction.

In the calculation we use the parameter values, the current quark mass $m_{q}\left(=m_{u}=m_{d}\right)=5 \mathrm{MeV}$ assuming $\mathrm{SU}(2)$ symmetry, and the free nucleon bag radius $R_{N}=0.8 \mathrm{fm}$, where both values are considered to be standard in QMC [17].

First, Fig. 1 shows ratios of the bound to free nucleon form factors calculated as a function of $Q^{2}$ for $\rho_{B}=\rho_{0}$ $=0.15 \mathrm{fm}^{-3}$ (the normal nuclear matter density) and $0.668 \rho_{0}$

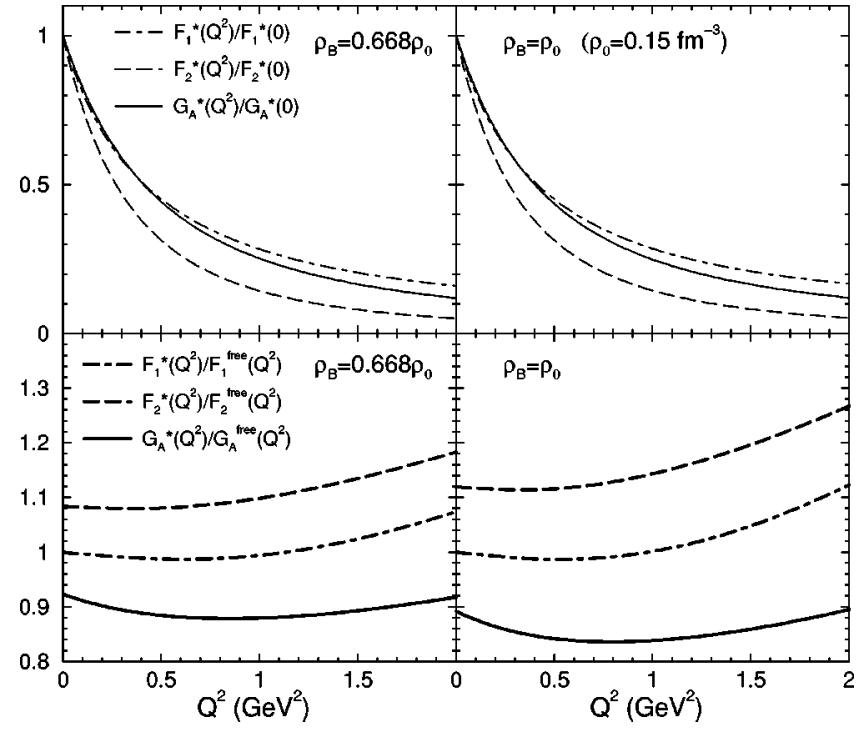

FIG. 1. Calculated ratios for the bound nucleon form factors.

(the Fermi momentum $k_{F}=225 \mathrm{MeV}$ for ${ }^{12} \mathrm{C}$ ). The lower panels in Fig. 1 show the enhancement of momentum dependence of $F_{2}^{*}\left(Q^{2}\right)$ and $G_{A}^{*}\left(Q^{2}\right)$, as well as the enhancement of $F_{2}^{*}(0)$ and quenching of $G_{A}^{*}(0)[9,14,24]$. Although the modification of the $Q^{2}$ dependence is small, we emphasize that this effect originates from the nucleon internal structure change. The main origin of this new $Q^{2}$ dependence is the Lorentz contraction effect to the quark wave function amplified by the reduced effective nucleon mass. [See also Eq. (3).] Note that the relative change of the bound nucleon form factor $F_{2}^{*}\left(Q^{2}\right)\left[G_{E}^{p^{*}}\left(Q^{2}\right)\right]$ to that of the free nucleon is an enhancement (quenching [9]) of $\sim 8 \%[4 \%]$ in ${ }^{12} \mathrm{C}$ at $Q^{2}$ $=0.15 \mathrm{GeV}^{2}$, and we are focusing on such relative change.

Next, we investigate the effect of the bound nucleon form factors on charged-current neutrino-nucleus scattering. We compute the inclusive ${ }^{12} \mathrm{C}\left(\nu_{\mu}, \mu^{-}\right) X$ differential and total cross sections, which have been measured by the liquid scintillator neutrino detector (LSND) collaboration [10]. We use the formalism described in Ref. [12], and that the empirical parametrizations of the electromagnetic [12,22] and axial [14,23] form factors for the free nucleon. [See Eq. (4).] A relativistic Fermi gas model is used implementing the bound nucleon form factors to calculate the differential cross section $\left\langle d \sigma / d E_{\mu}\right\rangle$, averaged over the LSND muon neutrino spectrum $\Phi\left(E_{\nu_{\mu}}\right)$ [12] for the full range of the LSND experimental spectrum [10], $0 \leqslant E_{\nu_{\mu}} \leqslant 300 \mathrm{MeV}$,

$$
\begin{aligned}
& \left\langle d \sigma / d E_{\mu}\right\rangle \\
& \quad=\left[\int_{0}^{\infty}\left(d \sigma / d E_{\mu}\right) \Phi\left(E_{\nu_{\mu}}\right) d E_{\nu_{\mu}}\right] /\left[\int_{0}^{\infty} \Phi\left(E_{\nu_{\mu}}\right) d E_{\nu_{\mu}}\right] .
\end{aligned}
$$

Figure 2 shows the result of $\left\langle d \sigma / d E_{\mu}\right\rangle$ calculated using the nucleon masses $m_{N}$ and $m_{N}^{*}$. For the Fermi momentum $k_{F}=225 \mathrm{MeV}\left(\rho_{B}=0.668 \rho_{0}\right)$ for ${ }^{12} \mathrm{C}$, we use the QMC calculated value, $m_{N}^{*}=802.8 \mathrm{MeV}$. A moderate quenching of the 


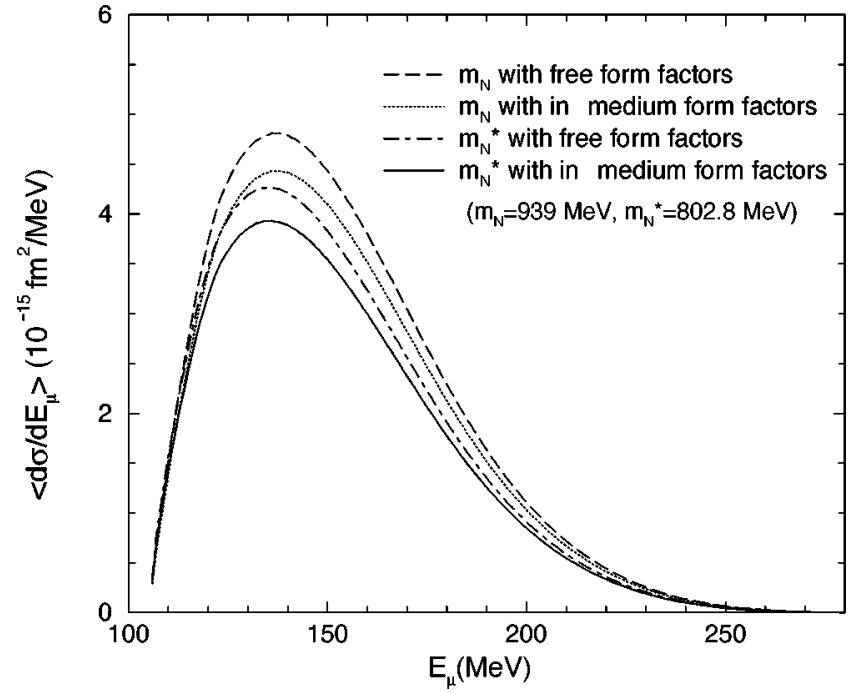

FIG. 2. Angle-integrated inclusive ${ }^{12} \mathrm{C}\left(\nu_{\mu}, \mu^{-}\right) X$ differential cross section as a function of the emitted muon energy $E_{\mu}$ using $E_{B}=0$ for all cases.

cross section can be observed due to the in-medium form factors for both cases. Although the effective nucleon mass can account for, to some extent, the binding effect (the Hugenholtz-van Hove theorem [25]), there is an alternative to include the binding effect, i.e., the "binding energy" $E_{B}$ is introduced and the available reaction energy $E$ is replaced by $E-E_{B}$. In this case, we use the free nucleon mass in the calculation. Since $E_{B}$ is an effective way of accounting for the binding effect [26], we regard $E_{B}$ as a parameter and perform calculations for $E_{B}=20,25$, and $30 \mathrm{MeV}$. (E.g., $E_{B}=25-27 \mathrm{MeV}$ is commonly used for the ${ }^{16} \mathrm{O}$ nucleus [27].) We emphasize that our aim is not to reproduce the LSND data, but to estimate the corrections due to the bound nucleon form factors. In Figs. 3 we present the results of $\left\langle d \sigma / d E_{\mu}\right\rangle$ for $E_{B}=20,25$, and $30 \mathrm{MeV}$. In both Figs. 2 and

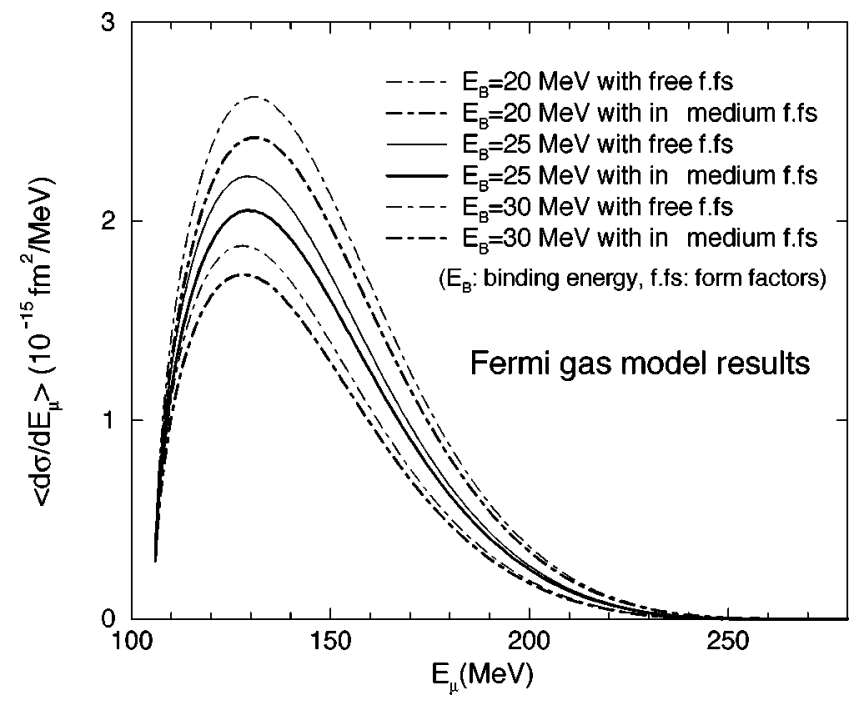

FIG. 3. Same as Fig. 2, but using $m_{N}=939 \mathrm{MeV}$ for all cases.
TABLE I. Calculated total cross sections for ${ }^{12} \mathrm{C}\left(\nu_{\mu}, \mu^{-}\right) X$. See the text for notations.

\begin{tabular}{llcc}
\hline \hline Notation & Type of calculation & $E_{B}(\mathrm{MeV})$ & $\langle\sigma\rangle$ in $10^{-40} \mathrm{~cm}^{2}$ \\
\hline$\langle\sigma(F, G)\rangle$ & $m_{N}, F_{1,2}\left(Q^{2}\right), G_{A, P}\left(Q^{2}\right)$ & 0 & 32.5 \\
$\left\langle\sigma\left(F^{*}, G^{*}\right)\right\rangle$ & $m_{N}, F_{1,2}^{*}\left(Q^{2}\right), G_{A, P}^{*}\left(Q^{2}\right)$ & 0 & 30.0 \\
$\langle\sigma(F, G)\rangle$ & $m_{N}^{*}, F_{1,2}\left(Q^{2}\right), G_{A, P}\left(Q^{2}\right)$ & 0 & 28.4 \\
$\left\langle\sigma\left(F^{*}, G^{*}\right)\right\rangle$ & $m_{N}^{*}, F_{1,2}^{*}\left(Q^{2}\right), G_{A, P}^{*}\left(Q^{2}\right)$ & 0 & 26.2 \\
& & & \\
$\left\langle\sigma\left(F^{*}, G\right)\right\rangle$ & $m_{N}, F_{1,2}^{*}\left(Q^{2}\right), G_{A, P}\left(Q^{2}\right)$ & 0 & 33.5 \\
$\left\langle\sigma\left(F, G^{*}\right)\right\rangle$ & $m_{N}, F_{1,2}\left(Q^{2}\right), G_{A, P}^{*}\left(Q^{2}\right)$ & 0 & 29.1 \\
& & & \\
$\langle\sigma(F, G)\rangle$ & $m_{N}, F_{1,2}\left(Q^{2}\right), G_{A, P}\left(Q^{2}\right)$ & 20 & 16.1 \\
$\left\langle\sigma\left(F^{*}, G^{*}\right)\right\rangle$ & $m_{N}, F_{1,2}^{*}\left(Q^{2}\right), G_{A, P}^{*}\left(Q^{2}\right)$ & 20 & 14.8 \\
$\langle\sigma(F, G)\rangle$ & $m_{N}, F_{1,2}\left(Q^{2}\right), G_{A, P}\left(Q^{2}\right)$ & 25 & 13.2 \\
$\left\langle\sigma\left(F^{*}, G^{*}\right)\right\rangle$ & $m_{N}, F_{1,2}^{*}\left(Q^{2}\right), G_{A, P}^{*}\left(Q^{2}\right)$ & 25 & 12.2 \\
$\langle\sigma(F, G)\rangle$ & $m_{N}, F_{1,2}\left(Q^{2}\right), G_{A, P}\left(Q^{2}\right)$ & 30 & 10.7 \\
$\left\langle\sigma\left(F^{*}, G^{*}\right)\right\rangle$ & $m_{N}, F_{1,2}^{*}\left(Q^{2}\right), G_{A, P}^{*}\left(Q^{2}\right)$ & 30 & 9.9 \\
& & & $10.6 \pm 0.3 \pm 1.8$ \\
& Experiment $[10](2002)$ & & $8.3 \pm 0.7 \pm 1.6$ \\
\hline \hline & Experiment $[10](1997)$ & & \\
& Experiment $[10](1995)$ & & \\
\hline \hline
\end{tabular}

3 , the bound nucleon form factors reduce the differential cross section. In Fig. 3, as the binding energy $E_{B}$ increases, the peak position shifts downward for both cases with the free and bound nucleon form factors. The similar tendency due to $m_{N}^{*}$ is also seen in Fig. 2 .

The total cross section is given by integrating Eq. (5) over the muon energy. We denote the cross section calculated with the free (bound) nucleon form factors $F_{1,2}\left(Q^{2}\right)$ and $G_{A, P}\left(Q^{2}\right)$ $\left[F_{1,2}^{*}\left(Q^{2}\right)\right.$ and $\left.G_{A, P}^{*}\left(Q^{2}\right)\right]$ as $\langle\sigma(F, G)\rangle\left[\left\langle\sigma\left(F^{*}, G^{*}\right)\right\rangle\right]$. Thus, $\langle\sigma(F, G)\rangle$ calculated with $m_{N}$ and $E_{B}=0$ corresponds to the free Fermi gas model result. The results with $E_{B}=0$ and either $m_{N}$ or $m_{N}^{*}$ are listed in the top group rows in Table I. The LSND experimental data [10] are also shown in the bottom group rows in Table I. As expected [10], the free Fermi gas result overestimates the data by a factor of 3 . The results obtained using the bound nucleon form factors, with either $m_{N}$ or $m_{N}^{*}$, similarly overestimate the LSND data. In order to make discussions more quantitative, we define

$$
R(\delta \sigma) \equiv\left[\langle\sigma(F, G)\rangle-\left\langle\sigma\left(F^{*}, G^{*}\right)\right\rangle\right] /\langle\sigma(F, G)\rangle .
$$

For the total cross sections calculated with $\left(m_{N}, m_{N}^{*}\right)$ and $E_{B}=0$, we get $R(\delta \sigma)=(7.7,7.7) \%$, respectively. Thus, the correction due to the bound nucleon form factors to the total cross section is not sensitive to $m_{N}$ or $m_{N}^{*}$ in the case of $E_{B}$ $=0$.

Next, we investigate which bound nucleon form factor gives dominant corrections to the total cross section. We calculate the total cross section with $m_{N}$, using the free and bound form factors for two cases, $\left[F_{1,2}^{*}\left(Q^{2}\right)\right.$ and $\left.G_{A, P}\left(Q^{2}\right)\right]$ and $\left[F_{1,2}\left(Q^{2}\right)\right.$ and $\left.G_{A, P}^{*}\left(Q^{2}\right)\right]$. They are denoted by $\left\langle\sigma\left(F^{*}, G\right)\right\rangle$ and $\left\langle\sigma\left(F, G^{*}\right)\right\rangle$, respectively. The results are given in the 
middle group rows in Table I. Together with the results in the upper group rows in Table I, we obtain inequalities for the total cross sections calculated with $m_{N}$ and $E_{B}=0$ :

$$
\left\langle\sigma\left(F, G^{*}\right)\right\rangle\left\langle\langle \sigma ( F ^ { * } , G ^ { * } ) \rangle \left\langle\langle\sigma(F, G)\rangle<\left\langle\sigma\left(F^{*}, G\right)\right\rangle\right.\right. \text {. }
$$

This shows that the most dominant reduction is driven by the axial form factor, $G_{A}^{*}\left(Q^{2}\right)$. (The induced pseudoscalar form factor $G_{P}\left(Q^{2}\right)$ gives only a few percent contribution when calculated using all free form factors.) Furthermore, $F_{1,2}^{*}\left(Q^{2}\right)$ enhance the total cross section [mostly due to $F_{2}^{*}\left(Q^{2}\right)$ ] as can be seen from the lower panel in Fig. 1.

The total cross sections for $E_{B}=20,25$ and $30 \mathrm{MeV}$ are listed in the bottom group rows in Table I. The bound nucleon form factors for these cases also reduce the total cross section relative to those calculated with the free form factors. In addition, the results are rather sensitive to the values for $E_{B}$. However, we find $R(\delta \sigma)=(8.1,7.6,7.5) \%$ for $E_{B}=(20,25,30) \mathrm{MeV}$, respectively. Thus, the effect of the bound nucleon form factors to the reduction rate is again not sensitive to $E_{B}$.
To summarize, we have estimated the effect of the bound nucleon form factors arising from the nucleon internal structure change on the inclusive ${ }^{12} \mathrm{C}\left(\nu_{\mu}, \mu^{-}\right) X$ cross sections. We have used a relativistic Fermi gas model implementing the bound nucleon form factors calculated in the QMC model. The effect of the bound nucleon form factors for this reaction is a reduction of $\sim 8 \%$ for the total cross section. This $\sim 8 \%$ reduction (or an order of $10 \%$ for a heavier nucleus) should be taken into account additionally to the standard nuclearstructure corrections. To draw a more definite conclusion, it is essential to perform a more precise, elaborate calculation within the framework of the random-phase approximation [12], including the effect of bound nucleon form factors. However, even at the present stage, it is important to point out that the correction due to the in-medium form factors could be significant for a precise estimate of the chargedcurrent neutrino-nucleus cross section.

We would like to thank D. H. Lu for providing us with the improved cloudy bag model codes, and K. Kubodera, K. Nakayama, and R. Seki for useful discussions. K.T. was supported by the Forschungszentrum-Jülich, Contract No. 41445282 (COSY-058).
[1] G. E. Brown and M. Rho, Phys. Rev. Lett. 66, 2720 (1991); P. A. M. Guichon, Nucl. Phys. A680, 229c (2001).

[2] M. Arneodo, Phys. Rep. 240, 301 (1994); D. F. Geesaman, K. Saito, and A. W. Thomas, Annu. Rev. Nucl. Part. Sci. 45, 337 (1995); R. P. Bickerstaff and A. W. Thomas, J. Phys. G 15, 1523 (1989).

[3] A. Arima et al., Adv. Nucl. Phys. 18, 1 (1987); I. S. Towner, Phys. Rep. 155, 263 (1987); F. Osterfeld, Rev. Mod. Phys. 64, 491 (1992).

[4] K. Tsushima and D. O. Riska, Nucl. Phys. A549, 313 (1992).

[5] K. Kubodera, J. Delorme, and M. Rho, Phys. Rev. Lett. 40, 755 (1978); M. Kirchbach, D. O. Riska, and K. Tsushima, Nucl. Phys. A542, 616 (1992); I. S. Towner, ibid. A542, 631 (1992).

[6] J. Morgenstern and Z.-E. Meziani, Phys. Lett. B 515, 269 (2001); K. Saito, K. Tsushima, and A. W. Thomas, ibid. 465, 27 (1999).

[7] S. Malov et al., Phys. Rev. C 62, 057302 (2000); S. Dieterich et al., Phys. Lett. B 500, 47 (2001); R. D. Ransome, Nucl. Phys. A699, 360c (2002); S. Strauch et al., Phys. Rev. Lett. 91, 052301 (2003); E93-049 Collaboration, S. Strauch, nuclex/0308026.

[8] J. J. Kelly, Phys. Rev. C 60, 044609 (1999); J. M. Udias and J. R. Vignote, ibid. 62, 034302 (2000); J. M. Udias et al., Phys. Rev. Lett. 83, 5451 (1999).

[9] D. H. Lu et al., Phys. Lett. B 417, 217 (1998); Phys. Rev. C 60, 068201 (1999).

[10] L. B. Auerbach et al., Phys. Rev. C 66, 015501 (2002); C. Athanassopoulos et al., ibid. 56, 2806 (1997); M. Albert et al., ibid. 51, R1065 (1995).

[11] E. Kolbe, K. Langanke, and P. Vogel, Nucl. Phys. A652, 91 (1999); C. Volpe et al., Phys. Rev. C 62, 015501 (2000); A. C. Hayes and I. S. Towner, ibid. 61, 044603 (2000); S. K. Singh et al., ibid. 57, 2687 (1998); F. Krmpotić, A. Mariano, and A.
Samana, Phys. Lett. B 541, 298 (2002); Y. Umino and J. M. Udias, Phys. Rev. C 52, 3399 (1995); C. Maieon, M. C. Martinez, J. A. Caballero, and J. M. Udias, ibid. 68, 048501 (2003).

[12] Hungchong Kim, J. Piekarewicz, and C. J. Horowitz, Phys. Rev. C 51, 2739 (1995); nucl-th/9502041.

[13] C. J. Horowitz, Hungchong Kim, and J. Piekarewicz, Phys. Rev. C 48, 3078 (1993).

[14] D. H. Lu, A. W. Thomas, and K. Tsushima, nucl-th/0112001.

[15] Hungchong Kim, S. Schramm, and C. J. Horowitz, Phys. Rev. C 53, 3131 (1996).

[16] K. Tsushima, D. O. Riska, and P. G. Blunden, Nucl. Phys. A559, 543 (1993).

[17] P. A. M. Guichon, Phys. Lett. B 200, 235 (1989); P. A. M. Guichon et al., Nucl. Phys. A601, 349 (1996); K. Saito, K. Tsushima, and A. W. Thomas, ibid. A609, 339 (1996); Phys. Rev. C 55, 2637 (1997).

[18] E.g., see K. Tsushima, hep-ph/0206069; K. Tsushima et al. nucl-th/0301078.

[19] A. W. Thomas, Adv. Nucl. Phys. 13, 1 (1984); G. A. Miller, Int. Rev. Nucl. Phys. 1, 190 (1984).

[20] D. H. Lu, A. W. Thomas, and A. G. Williams, Phys. Rev. C 57, 2628 (1998).

[21] A. L. Licht and A. Pagnamenta, Phys. Rev. D 2, 1150 (1970); 2, 1156 (1970).

[22] M. J. Musolf and T. W. Donnelly, Nucl. Phys. A546, 509 (1992).

[23] T. Kitagaki et al., Phys. Rev. D 28, 436 (1983).

[24] K. Saito and A. W. Thomas, Phys. Rev. C 51, 2757 (1995).

[25] K. A. Brueckner, Phys. Rev. 110, 597 (1958); N. M. Hugenholtz and L. Van Hove, Physica (Amsterdam) 24, 363 (1958).

[26] See also A. Hotta et al., Phys. Rev. C 30, 87 (1984).

[27] T. Kuramoto et al., Nucl. Phys. A512, 711 (1990). 